\title{
Avaliação histomorfométrica e ultra-estrutural da mucosa do cólon menor eqüino submetido a distensão ${ }^{1}$
}

\author{
Rafael R. Faleiros ${ }^{2 *}$, Delphim G. Macoris ${ }^{3}$, Geraldo Eleno S. Alves ${ }^{2}$, Carlos Henrique \\ Câmara Saquetti ${ }^{4}$ e Antonio Carlos Alessi ${ }^{5}$
}

\begin{abstract}
Faleiros R.R., Macoris D.G., Alves G.E.S., Saquetti C.H.C. \& Alessi A.C. 2007. [Histomorphometric and ultrastructural evaluation of the mucosa of the equine small colon subjected to distention.] Avaliação histomorfométrica e ultra-estrutural da mucosa do cólon menor eqüino submetido à distensão. Pesquisa Veterinária Brasileira 27(9):383-387. Departamento de Clínica e Cirurgia Veterinária, Faculdade de Ciências Agrárias e Veterinárias, Universidade Estadual Paulista, Campus de Jaboticabal, Rodovia Carlos Tonanni Km 5, Jaboticabal, SP 14870-000, Brazil. E-mail: faleiros@ufmg.br

Recently it has been shown that experimental distention of the small colon of horses promotes reduction of microvascular circulation and inflammation of the seromuscular layer associated with neutrophil accumulation in the lungs. However this model was not sufficient to induce evident histophatological changes in the mucosal layer. The aim of this study was to evaluate the mucosa subjected to that model of small colon distention by histomorphometry and scan electronic microscopy (SEM). Sixteen horses were used. In the distended group (DG), nine of them were subjected to distention of the small colon by a surgically implanted intraluminal balloon that was inflated with a pressure of $40 \mathrm{~mm} \mathrm{Hg}$ during 4 hours. In the sham-operated group (SG), the balloon was implanted but not inflated. Full-thickness intestinal samples were collected before and after obstruction and after 1.5 and 12 hours of decompression. By SEM, it was observed that the mucosa turned flat and smooth after distention and returned to the wrinkled original appearance after decompression. Twelve hours after decompression the mucosa had a more irregular appearance with points of fragmentation. There was a reduction in mucosa thickness after distention, returning to basal values after decompression. Instead of the fact that there were changes in appearance and thickness, it was concluded that the mucosa could borne up the compression caused by distention returning to the original characteristics without major lesions.
\end{abstract}

INDEX TERMS: Small colon, intestinal distention, colic, horse.

\footnotetext{
${ }^{1}$ Recebido em 28 de junho de 2007.

Aceito para publicação em 15 de setembro de 2007.

Parte da Tese de Doutorado do primeiro autor, apresentada ao Programa de Pós-Graduação em Cirurgia Veterinária, Faculdade de Ciências Agrárias e Veterinárias (FCAV), Universidade Estadual Paulista (Unesp), Campus de Jaboticabal, SP.

${ }^{2}$ Depto Clínica e Cirurgia Veterinárias, Escola de Veterinária, Universidade Federal de Minas Gerais (UFMG), Av. Antonio Carlos 6627, Belo Horizonte, MG 31270-901, Brasil. *Autor para correspondência: faleiros@ufmg.br

${ }^{3}$ Depto Clínica e Cirurgia Veterinária, FCAV, Unesp-Jaboticabal, Rodovia Carlos Tonanni Km 5, Jaboticabal, SP 14870-000, Brasil.

${ }^{4}$ Faculdade de Medicina Veterinária, UPIS Faculdades Integradas, SEP SUL EQ 712/912, Conj. A, Brasília, DF 70390-125, Brasil.

${ }^{5}$ Depto Patologia, FCAV, Unesp-Jaboticabal, SP.
}

RESUMO.- Estudos recentes demonstraram que a distensão luminal do cólon menor, apesar de ter promovido redução da irrigação da camada seromuscular resultando em inflamação intensa, não acarretou em lesões histopatológicas aparentes na mucosa. $\mathrm{O}$ objetivo do presente estudo foi avaliar, por meio de histomorfometria e microscopia eletrônica de varredura (MEV), os efeitos da distensão intraluminal sobre a mucosa do cólon menor. Utilizaram-se 16 equiinos. No Grupo distendido (GD), nove deles foram submetidos a quatro horas de distensão intraluminal do cólon menor por um balão inflado com pressão de $40 \mathrm{~mm}$ de $\mathrm{Hg}$. No Grupo instrumentado (GI), o balão foi introduzido no lume, mas sem provocar distensão. Colheramse amostras intestinais antes e ao fim da obstrução e após 1,5 e 12 horas de reperfusão. À MEV observou-se em GD que a superfície da mucosa adquiriu um aspecto liso, aplainado, após 
a distensão, retornando ao aspecto rugoso após descompressão. Ao fim do experimento, a superfície da mucosa se apresentava com aspecto um pouco mais irregular, com pontos de fragmentação. À histomorfometria observou-se em GD uma redução da espessura da mucosa em relação ao controle após a distensão, retornando a valores semelhantes aos basais após descompressão. Concluiu-se que a mucosa, apesar de ter seu aspecto alterado e sua espessura reduzida, conseguiu suportar a distensão promovida pelo aumento de pressão intraluminal, retornando às suas características originais sem apresentar lesões significativas.

TERMOS DE INDEXAÇÃO: Cólon menor, distensão intestinal, cólica, eqüino.

\section{INTRODUÇÃO}

As obstruções intestinais têm sido apontadas como a principal causa de internação e óbitos de equiinos em hospitais veterinários por todo o mundo. Tais obstruções ocorrem pela oclusão do lume intestinal com ou sem comprometimento da irrigação local. Em ambos os tipos de obstrução ocorre aumento da pressão intraluminal pelo acúmulo de conteúdo intestinal sólido, líquido e gás nos segmentos orais à obstrução.

Vários estudos têm demonstrado, de formas clínica e experimental, que o aumento de pressão intraluminal, e conseqüente distensão, têm associação direta com o prognóstico e com complicações pós-operatórias como aderências (Lundin et al. 1989) e íleo paralítico (Malone \& Kannan 2001).

A distensão intestinal também pode ocorrer nos casos de compactação do conteúdo intestinal ou pela ação de concreções intestinais e corpos estranhos impelidos aboralmente. Este tipo de situação tem sido descrito com frequiência nas obstruções por enterólitos e corpos estranhos no cólon menor dos eqüinos.

Recentemente foi desenvolvido um modelo para simular este tipo de obstrução no cólon menor (Faleiros et al. 2002). Após quatro horas de distensão intraluminal, apesar de ter havido intensa inflamação na camada seromuscular associada ao acúmulo de neutrófilos nos pulmões, não se observaram lesões histopatológicas aparentes na mucosa (Faleiros et al. 2007). Com a hipótese de que uma avaliação mais detalhada poderia revelar alterações não observadas à microscopia convencional, o objetivo do presente estudo foi avaliar, por meio de histomorfometria e microscopia eletrônica de varredura (MEV), os efeitos da distensão intraluminal sobre a mucosa do cólon menor.

\section{MATERIAL E MÉTODOS}

\section{Animais}

Foram utilizados 16 eqüinos hígidos, cinco machos castrados e onze fêmeas não gestantes, com idade média de $7 \pm 3,1$ anos, pesando em média $351 \pm 54 \mathrm{~kg}$ e com escore corporal de 3 a 4 em uma escala de 1 a 5 (Speirs 1997), que foram divididos aleatoriamente em dois grupos. $\mathrm{O}$ grupo instrumentado (GI) com sete e o grupo distendido (GD) com nove animais. Os eqüinos foram desverminados (ivermectina, $200 \mu / \mathrm{kg}$ per os) e mantidos por 15 dias em piquete coletivo com suplementação à base de cinco quilos de feno e dois quilos de ração comercial por animal por dia. Este estudo foi aprovado pela Comissão de Ética e Bem Estar Animal da Faculdade de Ciências Agrárias da UNESP.

\section{Laparotomia em posição quadrupedal}

Após doze horas de jejum, os animais foram contidos em brete, sedados com xilazina $(0,5 \mathrm{mg} / \mathrm{kg}$ i.v.) e butorfanol $(0,1 \mathrm{mg} / \mathrm{kg}$ i.m.) e o flanco esquerdo foi anestesiado por meio da técnica de "L" invertido, utilizando associação (1:1) de lidocaína $2 \%$ e de bupivacaína $0,5 \%$. Através de laparotomia, um segmento do cólon menor foi exposto.

Após ordenha aboral do conteúdo intestinal, uma enterotomia de $5 \mathrm{~cm}$ foi realizada na margem anti-mesentérica do cólon menor, a aproximadamente $50 \mathrm{~cm}$ da transição para o cólon transverso, imediatamente após ao segmento intestinal irrigado pela primeiro ramo da artéria mesentérica caudal. Para promover a obstrução intestinal, foi inserido pela enterotomia, em direção oral, um balão de látex vazio. Para ocluir a abertura desse balão, foi montada uma rolha de poliamida com um orifício central onde havia acoplado um equipo de soro com uma pêra insufladora em sua extremidade. Para evitar o deslocamento do balão em direção oral-aboral foram colocadas, em ambas extremidades do segmento experimental, ligaduras frouxas feitas com dreno de Penrose $n^{\circ}=3$, aplicadas ao redor do cólon menor de modo a não ocluir a irrigação mesentérica e mural. Essas ligaduras foram fixadas na margem anti-mesentérica com um ponto simples, em padrão " $x$ ". A enterorrafia foi realizada em plano simples contínuo com fio de polipropileno calibre 00 , deixando sair o equipo por entre as bordas da ferida.

Em nove animais, $o$ balão foi inflado com ar até atingir a pressão de $40 \mathrm{~mm}$ de $\mathrm{Hg}$, correspondendo ao Grupo Distendido (GD). Em outros sete animais, o balão foi apenas preenchido com ar, mas sem resultar em pressão positiva e qualquer distensão do lume intestinal, caracterizando-se assim o Grupo Instrumentado (GI). O cólon menor foi reposicionado na cavidade peritoneal e a parede abdominal foi suturada por meio de padrão simples contínuo com fio de poliamida calibre 0,60 , deixando também sair o equipo por entre as bordas da ferida. Esse equipo foi acoplado a um transdutor de pressão (P 23XL, Ohmeda, EUA) montado em fisiógrafo (1-8P-24;5, Grass Instruments, EUA), devidamente calibrado, por onde foi registrada a pressão no interior do balão. Em GD a pressão intraluminal era aferida nos primeiros dez minutos e, após, em intervalos de 30 minutos, sendo que, se necessário fosse, efetuava-se a insuflação ou retirada de ar do balão, para que a pressão se mantivesse em $40 \mathrm{~mm}$ de $\mathrm{Hg}$.

\section{Anestesia geral}

Ao final de 3 horas, os animais receberam pré-anestesia (MPA) com midazolam $(0,15 \mathrm{mg} / \mathrm{kg}$ i.v.), seguida por infusão sob pressão de éter gliceril guaiacol a $10 \%$ até que decúbito lateral fosse atingido, quando a anestesia foi induzida com tiopental (3mg/kg i.v.). Os animais foram posicionados em decúbito dorsal e durante a manutenção anestésica administrou-se de $2 \%$ a $3 \%$ de halotano, por meio de vaporizador compensado (Tec 3 Style, Vaporizer Sales \& Service Inc, EUA). A pressão arterial média (PAM) foi monitorada através de cateter, inserido no ramo mandibular da artéria facial e conectado, por meio de uma extensão preenchida com solução salina fisiológica heparinizada (20 UI/ml), a um manômetro aneróide. Para manutenção da PAM entre 70 e $100 \mathrm{~mm}$ de $\mathrm{Hg}$ foram administrados de $5-10 \mathrm{ml} / \mathrm{kg} / \mathrm{h}$ (i.v.) de solução de Ringer lactato. Quando a fluidoterapia não foi suficiente para tal, infundiu-se de $1-2 \mu \mathrm{g} / \mathrm{kg} / \mathrm{min}$ (i.v.) de dobutamina. Amostras de sangue arterial foram coletadas de 30 em 30 minutos para análise gasométrica (i-Stat PCA, i-Stat Co, EUA). Durante toda a anestesia utilizou-se de ventilação controlada, para a manutenção das pressões parciais de $\mathrm{O}_{2}>100 \mathrm{~mm}$ de $\mathrm{Hg}$ e de $\mathrm{CO}_{2}<50 \mathrm{~mm}$ de $\mathrm{Hg}$. 


\section{Laparotomia em decúbito dorsal}

Estabilizada a anestesia, realizou-se celiotomia através de incisão mediana, de aproximadamente $25 \mathrm{~cm}$, para permitir a exposição do cólon menor distendido. Completado o período de 4 horas após a indução da obstrução, promoveu-se a desobstrução do lume por meio do esvaziamento do balão e de sua retirada. Após enterorrafia, o segmento de cólon menor foi retornado para a cavidade abdominal onde foi reperfundido por um período de 90 minutos. Após esse período de reperfusão, realizou-se a laparorrafia segundo técnica de rotina e foi permitida a recuperação anestésica.

No início do período de recuperação, os animais receberam 0,005 $\mathrm{mg} / \mathrm{kg}$ de buprenorfina por via intramuscular, com intuito de promover analgesia. Doze horas após a desobstrução intestinal, os animais foram sedados com xilazina ( $1 \mathrm{mg} / \mathrm{kg}$ i.v.) e acepromazina $(0,1 \mathrm{mg} / \mathrm{kg}$ i.v.), anestesia geral foi induzida com tiopental $(3 \mathrm{mg} / \mathrm{kg})$ administrado na artéria carótida e procedeu-se a eutanásia por infusão intravenosa de solução saturada de sulfato de magnésio.

\section{Colheitas de amostras}

Como testemunhas, foram colhidas amostras do cólon menor no momento da enterotomia para colocação do balão. Também foram colhidas amostras intestinais no segmento experimental ao final do período de obstrução e após 1,5 e 12 horas após a remoção do balão. Ao final do experimento, também foram colhidas uma amostra de cólon menor em segmento aboral, distante cerca de um metro do local da obstrução, e de uma a três amostras dos pulmões colhidas em lobos distintos. As amostras intestinais foram constituídas de fragmentos de intestino com aproximadamente $2 \mathrm{x}$ $5 \mathrm{~cm}$, colhidos na margem anti-mesentérica paralelos à tênia, por meio de uma incisão transversal com o bisturi incidindo todas as camadas. Após remoção de cada fragmento, a incisão foi suturada usando-se fio de poliglactina $910(00)$, em padrão simples contínuo seguido de sutura invaginante do tipo Cushing.

As amostras intestinais foram dividas em dois fragmentos. $\mathrm{O}$ primeiro foi imediatamente fixado em solução de formalina tamponada a $10 \%$ e, posteriormente, processado segundo técnicas rotineiras de inclusão em parafina, cortadas em micrótomo a cinco $\mathrm{mm}$, coradas pela hematoxilina e eosina (H-E) (Luna, 1968) e examinadas em microscópio de luz. O segundo fragmento foi fixado em solução de glutaraldeído tamponado e armazenado a $4^{\circ} \mathrm{C}$, para posterior processamento de rotina para microscopia eletrônica de varredura.

\section{Análise histomorfológica}

Para análise quantitativa, foi mensurada a espessura da mucosa. Para tanto as imagens obtidas com objetiva de $4 \mathrm{x}$ foram digitalizadas e analisadas por programa de análise de imagens (Image Pro Plus 3.0, Media Cybernetics, EUA), considerando como amostra representativa uma área de no mínimo $10 \%$ da extensão da lâmina.

\section{Análise ultra-estrutural}

As amostras intestinais fixadas em glutaraldeído foram cortadas em fragmentos com aproximadamente $16 \mathrm{~mm}^{2}$, tratadas com tetróxido de ósmio ( $2 \%$ ) por duas horas e, posteriormente, desidratadas por passagens consecutivas de $15 \mathrm{~min}$ em concentrações crescentes de álcool etílico. Após secagem em $\mathrm{CO}_{2}$ sob pressão, por meio de Secador de Ponto Crítico (EMS 850, EMS, USA), os fragmentos foram metalizados em Metalizador de Fluxo Iônico (Denton Vacuum Desk II, Denton Vaccum, USA) por período de 70 segundos em potência de $70 \mathrm{~mA}$. A mucosa foi examinada em Microscópico Eletrônico de Varredura (JSM-5410, JEOL, Japão), operando em 15kV em um aumento de 750 vezes. Foram analisadas e fotografadas as amostras de três animais de cada grupo com o intuito de descrever e registrar possíveis alterações morfológicas.

\section{Análise de dados}

As avaliações estatísticas paramétricas foram feitas através de análise de variância em blocos ao acaso e submetidas ao teste de Student-Newman-Keuls para comparação tempos dos em um mesmo grupo. $O$ teste $t$ de Student foi utilizado para comparação de grupos em cada tempo (Sampaio 1998). Foi considerado nível de significância de P£0,05.

\section{RESULTADOS}

As medidas da espessura da mucosa estão representadas na Figura 1. Nas amostras controles as médias $( \pm D P)$ foram $588 \pm 53 \mu \mathrm{m}$ em GI e $562 \pm 95 \mu \mathrm{m}$ em GD, não havendo diferença entre elas. Observou-se em GD uma redução da espessura da mucosa em relação aos valores do controle, com retorno a valores equivalentes 1,5 horas após a descompressão. Essa alteração não foi observada em GI.

Pela microscopia eletrônica de varredura (MEV), de acordo com a técnica e aumentos utilizados, foi possível observar que em GD a superfície da mucosa, anteriormente rugosa, adquiriu um aspecto liso, aplainado, após a distensão (Fig.2). Após descompressão, a mucosa retomou o aspecto rugoso, porém mais abaulado que o inicial. Ao fim do experimento, a superfície da mucosa se apresentava com aspecto mais irregular, com alguns pontos de fragmentação.

\section{DISCUSSÃO}

A análise ultra-estrutural de GD mostrou que a mucosa perdeu sua rugosidade característica ao final da distensão, adquirindo um aspecto achatado (Fig.2). Este achado está certamente associado à ação mecânica do balão e coincide com a redução significativa da espessura da mucosa (Fig.1). A microscopia eletrônica por varredura também revelou em GD que, após a reperfusão, havia alguns pontos de fragmentação

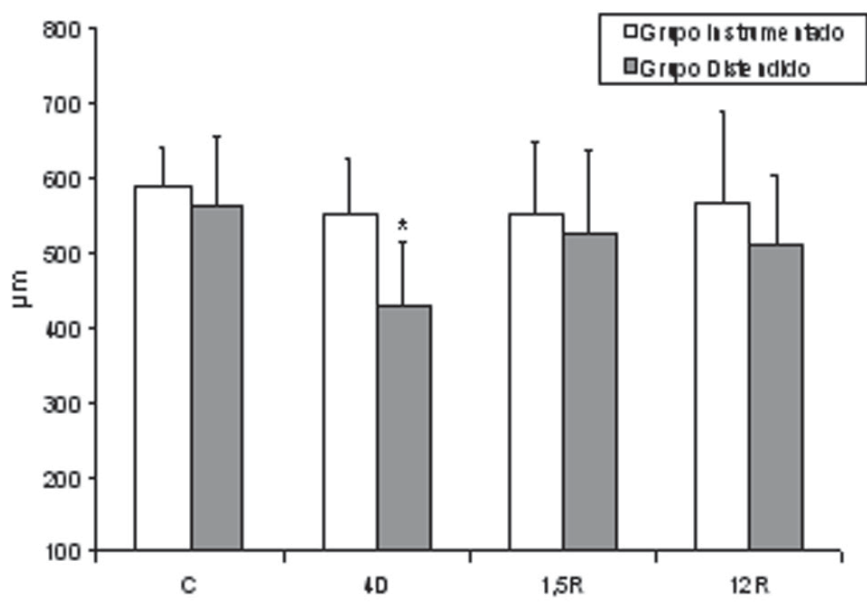

Fig.1. Médias (M) e desvios padrão (DP) da espessura de mucosa em amostras controle $(C)$, após 4 horas de obstrução experimental (4D) e após 1,5 h $(1,5 R)$ e 12 h $(12 R)$ de reperfusão. *Em um mesmo grupo, difere da média das amostras do controle, $\mathrm{P}<0,05$. 

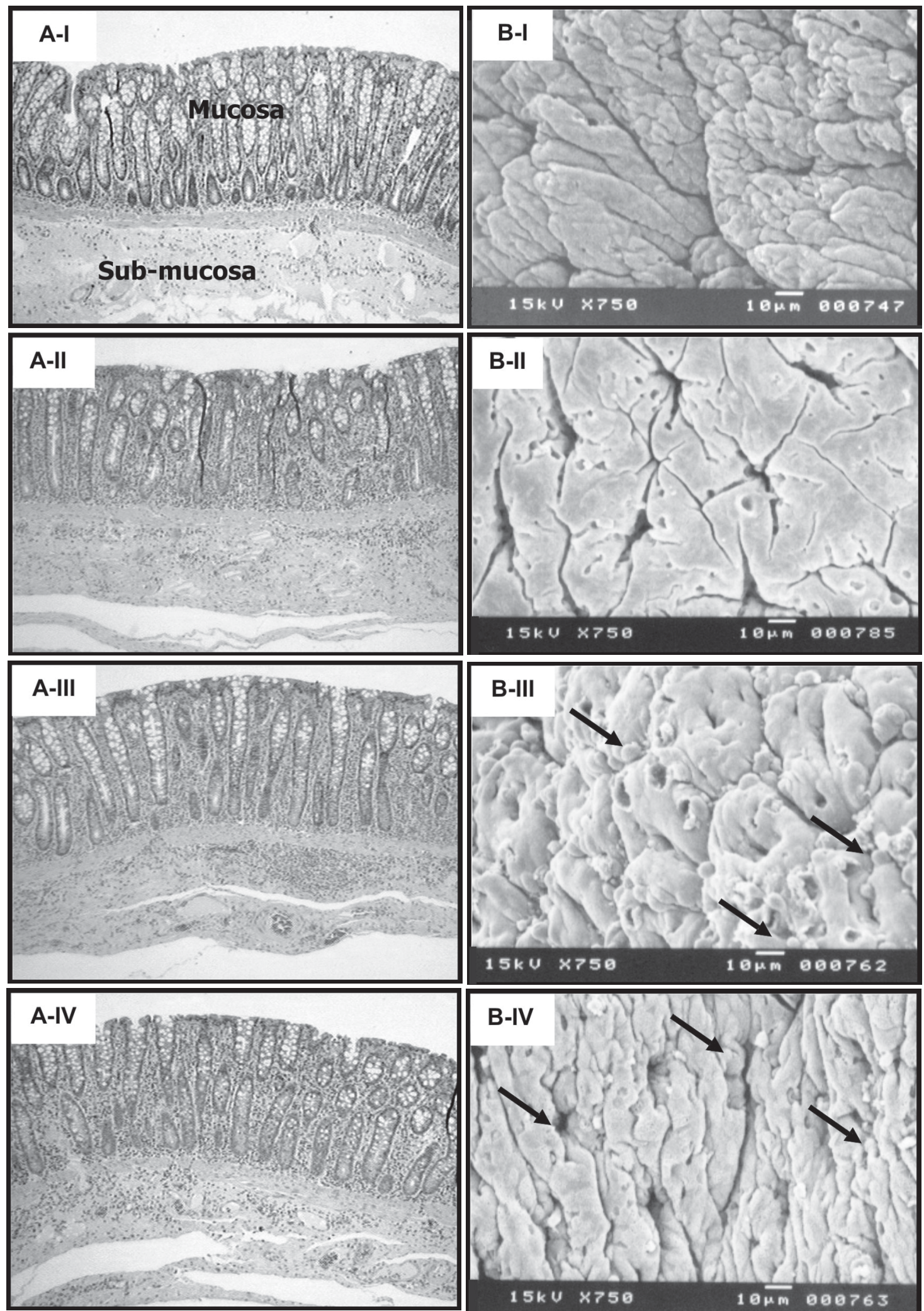

Fig.2. Fotomicrografias (A) e eletromicrografias de varreduras (B) do cólon menor equïno de animais do Grupo Distendido. Antes da obstrução (I), ao final de 4 horas de distensão (II) e após 1,5 (III) e 12 horas (IV) de reperfusão. Observar que ao final da distensão, a mucosa apresentou espessura reduzida (A-II) e superfície de aspecto aplainado (B-II). Após 1,5 horas de reperfusão a superfície da mucosa apresenta aspecto abaulado (B-III). Nas amostras colhidas após a reperfusão, observaram-se alguns pontos de fragmentação na superfície da mucosa, não evidenciados à histopatologia em (A) H-E, obj.4x, e (B) 15kV, 750x. 
do tecido. Contudo, esta lesão não se evidenciou nos cortes histológicos convencionais (Fig.2).

A compressão da parede do cólon menor, com pressão intraluminal de $40 \mathrm{~mm}$ de $\mathrm{Hg}$, apesar de ter sido capaz de reduzir a perfusão microvascular para $26,4 \%$ (Faleiros et al. 2002) e de promover alterações significativas no líquido peritoneal em um período de três horas (Faleiros 2003), não foi suficiente para promover desvitalização irreversível do cólon menor, após quatro horas de distensão e doze de reperfusão. No presente trabalho, o intuito foi produzir uma situação de compressão direta da parede intestinal por enterólito, ou corpo estranho, no terço oral do cólon menor. Esta pressão foi utilizada, pois, além de se mostrar suficiente para provocar isquemia microvascular, estava acima de $38 \mathrm{~cm}$ de $\mathrm{H}_{2} 0(28,1 \mathrm{~mm}$ de $\mathrm{Hg}$ ), pressão determinada como limite mínimo ideal para predizer prognóstico desfavorável em casos clínicos de distensão do cólon maior de eqüinos (Moore et al. 1995). O tempo de quatro horas foi utilizado com base em estudo anterior, em que três horas de isquemia arteriovenosa completa, seguidas de 1,5 horas de reperfusão, foram suficientes para provocar degeneração extensa na mucosa do cólon menor (Faleiros et al. 2001). O cólon menor mostrou-se assim mais resistente ao aumento de pressão intraluminal do que o cólon maior e mais resistente à isquemia microvascular causada por compressão intraluminal da parede intestinal, do que a isquemia por oclusão dos vasos mesentéricos e murais.

A predominância de lesões na camada seromuscular do cólon menor foi um fato esperado, uma vez que já havia sido demonstrado, por meio de microesferas (Faleiros et al. 2002), que a redução de perfusão ocorre predominantemente nessa camada. Experimentos realizados com distensão intraluminal do jejuno de eqüinos também revelaram lesões discretas na mucosa e lesões mais evidentes na seromuscular (Allen et al. 1988, Lundin et al. 1989, Parks et al. 1989, Dabareiner et al. 1993, 2001a,b). Este achado poderia ser explicado pelo tipo de constituição tecidual das camadas. A seromuscular é constituída essencialmente de tecido muscular com pouco tecido conjuntivo, possuindo assim reduzida capacidade de se distender frente ao aumento de pressão luminal. Por outro lado, a submucosa possui mais tecido conjuntivo frouxo, o que permitiria melhor acomodação da mucosa e conseguinte distribuição e transmissão das forças advindas do lume. Contudo, experimentos realizados com distensão em suínos (Ruf et al. 1980) e cães (Shitaka et al. 1983) verificaram maior redução de perfusão na submucosa e mucosa em relação à seromuscular. Assim, a ausência de lesões na mucosa do cólon menor distendido corrobora para a hipótese levantada por Faleiros et al. (2002), de que existe no eqüino um mecanismo de proteção dessa camada nos casos de compressão da parede intestinal, devido ao aumento da pressão intraluminal.

\section{CONCLUSÕES}

Concluiu-se que a mucosa, apesar de ter seu aspecto alterado e sua espessura reduzida, conseguiu suportar a distensão promovida pelo aumento de pressão intraluminal, retornando às suas características originais sem apresentar lesões signifi- cativas. A ausência de lesões significativas na mucosa está de acordo com os achados prévios sobre a distribuição da perfusão sanguiínea na parede (Faleiros et al. 2002), mostrando que pode haver um mecanismo de proteção à mucosa nos casos de distensão intraluminal do cólon menor eqüino.

Agradecimentos.- À FAPESP (Proc.00/02151-6) e à CAPES/PICDT, pelo apoio financeiro. Ao Laboratório de Imunopatologia do Departamento de Patologia da FCAV, Unesp-Jaboticabal, pelo auxílio na histomorfometria.

\section{REFERÊNCIAS}

Allen D., White N.A. \& Tyler D.E. 1988. Morphologic effects of experimental distention of equine small intestine. Vet. Surg. 17:10-14.

Dabareiner R.M., Sullins K.E., Snyder J.R., White N.A. \& Gardner I.A. 1993. Evaluation of the microcirculation of the equine small intestine after intraluminal distention and subsequent decompression. Am. J. Vet. Res. 54:1673-1682.

Dabareiner R.M., Sullins K.E., White N.A. \& Snyder J.R. 2001a. Serosal injury in the equine jejunum and ascending colon after ischemia-reperfusion or intraluminal distention and decompression. Vet. Surg. 30:114-125.

Dabareiner R.M., White N.A. \& Donaldson L.L. 2001b. Effects of intraluminal distention and decompression on microvascular permeability and hemodynamics of equine jejunum. Vet. Surg. 30:114-125.

Faleiros R.R. 2003. Obstrução experimental do cólon menor eqüino: aspectos clínicos, patológicos e terapêuticos. Tese de Doutorado em Cirurgia Veterinária, Faculdade de Ciências Agrárias e Veterinária, Unesp-Jaboticabal. 253p.

Faleiros R.R., Alves G.E.S., Santos R.L., Marques Jr A.P. \& Macoris D.G. 2001. Experimental ischemia and reperfusion in equine small colon. Arq. Bras. Med. Vet. Zootec. 53:341-350.

Faleiros R.R., Macoris D.G., Alessi A.C., Saquetti C.H. \& Rasera L. 2002. Effect of intraluminal distention on microvascular perfusion in the equine small colon. Am. J. Vet. Res. 63:1292-1297.

Faleiros R.R., Macoris D.G., Alves G.E.S., Souza D., Teixeira M.M. \& Moore R.M. 2007. Local and remote lesions in horses subjected to small colon distention and decompression. Can. J. Vet. Res. (Em publicação)

Luna, L.G. 1968. Manual of Histologic Staining Methods of the Armed Forces Institute of Pathology. $3^{\text {rd }}$ ed. McGraw Hill, New York.

Lundin C., Sullins K.E., White N.A., Clem M.F., Debowes R.M. \& Pfeiffer C.A. 1989. Induction of peritoneal adhesions with small intestinal ischaemia and distention in the foal. Equine Vet. J. 21:451-458.

Malone E.C. \& Kannan M.S. 2001. Effects of intestinal ischemia on in vitro activity of adjacent jejunum in samples obtained from ponies. Am. J. Vet. Res. 62:1973-1977.

Moore R.M., Muir W.W. \& Granger D.N. 1995 Mechanisms of gastrointestinal ischemia-reperfusion injury and therapeutic interventions: a review and its implications in the horse. J. Vet. Intern. Med. 9:115-132.

Parks A.H., Stick J.A., Arden W.A., Chou C.C. \& Hengemuhle S.M. 1989. Effects of distention and neostigmine on jejunal vascular resistance, oxygen uptake, and intraluminal pressure changes in ponies. Am. J. Vet. Res. 50:5458.

Ruf W., Suehiro G.T., Suehiro A., Pressler V. \& McNamara J.J. 1980. Intestinal blood flow at various intraluminal pressures in the piglet closed abdomen. Ann. Surg. 191:157-163.

Sampaio I.B.M. 1998. Delineamento em blocos ao acaso, p.50-60. In: Idem (ed.), Estatística Aplicada à Experimentação Animal. Editora FEP-MVZ, Belo Horizonte.

Shikata J., Shida T., Amino K. \& Ishioka K. 1983. Experimental studies on the hemodynamics of the small intestine following increased intraluminal pressure. Surg. Gynec. Obst. 156:155-160.

Speirs V.C. 1997. The alimentary tract, p.261-298. In: Idem (ed.), Clinical Examinations of Horses. W.B. Saunders, Philadelphia. 